\title{
Chronique des travaux universitaires
}

Jean-Pierre Watté

\section{CpenEdition}

Journals

Édition électronique

URL : http://journals.openedition.org/rao/738

DOI : $10.4000 /$ rao.738

ISBN : 978-2-7535-1608-3

ISSN : 1775-3732

Éditeur

Presses universitaires de Rennes

Édition imprimée

Date de publication : 20 décembre 2008

Pagination : 343-344

ISBN : 978-2-7535-0789-0

ISSN : 0767-709X

\section{Référence électronique}

Jean-Pierre Watté, "Chronique des travaux universitaires », Revue archéologique de l'Ouest [En ligne],

25 | 2008, mis en ligne le 20 décembre 2008, consulté le 06 décembre 2020. URL : http://

journals.openedition.org/rao/738; DOI : https://doi.org/10.4000/rao.738 


\title{
Chronique des travaux universitaires
}

\author{
Jean-Pierre WATTÉ
}

Nous continuons ici la recension des thèses et mémoires d'archéologie ou de disciplines connexes soutenues dans l'Ouest ainsi que celle des travaux concernant à un titre ou à un autre l'archéologie du "Grand-Ouest » français, selon la formule initiée dans les précédents volumes de la $R A O$.

Compte tenu de la difficulté matérielle à obtenir un dépouillement exhaustif en la matière, la rédaction de la $R A O$ remercie chaleureusement par avance celles et ceux (notamment les lauréats et leurs directeurs de recherche) qui voudront bien lui faire part des références susceptibles de figurer utilement dans cette chronique, quel que soit le cadre - français ou étranger - dans lequel ces travaux auront été conduits et, par là même, contribuer à les faire connaître.

\section{Université de RenNes 1 - BeAulieu}

\section{Mémoires de Master 2}

Dartors, Vincent (2008) - Composition élémentaire des objets en bronze de l'ouest de la France à l'âge du Bronze. Approche diachronique et spatiale de la métallurgie atlantique de l'àge du Bronze. (dir. J. Gomez de Soto, B. Mille et $S$. Boulud).

LEFORT, Anthony (2008) - Les relations trans-manche à la fin de l'âge du Fer : contribution et perspectives à travers l'étude du site d'Urville-Nacqueville (Manche). 3 vol., 101 p., 106 fig., 11 pl., annexe 200 p. (direction. S. Wirth; tuteurs scientifiques C. Marcigny et I. Ralston).

\section{Thèse de doctorat}

Matay, Christophe (2008) - Les céramiques peintes non tournées dans le centre-ouest de la France et ses marges, de l'àge du Bronze à La Tène ancienne. Apport à la connaissance des cultures matérielles de l'ouest de la France. (dir. J.-P. Pautreau).

\section{Université de Rennes 2 - Haute-Bretagne}

\section{Mémoires de Master 1}

Praud, Fanny (2008) - Les dépôts de l'Âge du Fer dans les milieux humides. (dir. J. Gomez de Soto).

Villemin, Alizée (2008) - Le Cheval celte. Synthèse sur les tombes à restes de chevaux en France pour l'Âge du Fer, et tentative d'interprétation. (dir. J. Gomez de Soto). [traite de la Normandie]

SetzKorn, Caroline (2008) - Conceptualisation spatiale des pratiques funéraires de La Tène moyenne et de la Tène finale en Normandie. 2 vol. 105 p. et inventaire, (dir. J. Gomez de Soto et C. Marcigny).

\section{UNIVERSITÉ DE RENNES 2 - UNIVERSITÉ DE NANTES}

\section{Mémoire de Master 1}

Dubuquoy, Julien (2008) - Le dépôt de Keriéro à Bangor (Belle-Île, Morbihan) : étude des pièces d'armement. (dir. S. Boulud). 


\section{Université de Paris 1 - Panthéon-Sorbonne}

\section{Mémoires de Master 1}

BurtTe, Hélène (2008) - Analyse spatiale des dépôts non funéraires du Massif Armoricain, de 1600 à 450 av. J.-C. environ. 2 vol., 37 p et 35 cartes. (dir. P. Brun).

Bonelli, Laetitia (2008) - Inventaire et étude des enduits peints des thermes $d u$ Vieil-Evreux (Eure). 3 vol. : texte 137 p., 100 fig., inventaire. (dir. F. Dumasy, L. Guyard et F. Monnier).

Fontaine, Christiane (2008) - Le Vieil-Évreux sous le prisme des archives. 2 vol. : texte 205 p., corpus 66 p., (dir. F. Dumasy et L. Guyard).

Gandois, Henri (2008) - L'apparition du bronze à l'étain dans le nord-ouest de la France. (dir. P. Brun).

Hartz, Cécile (2007) - L'habitat urbain à Évreuxe à l'époque gallo-romaine. Milieu du Ir $^{r}$ siècle av. J.-C. - - viècle. 2 vol. : Texte, 265 p.; Planches et annexes. (dir. F. Dumasy et L. Guyard).

Lemerle, Jean-Baptiste (2007) - Les palestres en Gaule romaine. (dir. F. Dumasy) [concerne Le Viel-Évreux].

\section{Mémoires de Master 2}

Hartz, Cécile (2008) - Habitat et trame urbaine dans les agglomérations secondaires de la Cité des Aulerques Eburovices. 2 vol. : 1-Texte et illustrations, 317 p.; 2Annexes. (direction F. Dumasy et L. Guyard).

Moreau, Julie (2008) - Multifonctionnalité des haches métalliques de l'âge du Bronze dans la moitié nord de la France. (dir. Patrice Brun).

Zeller, Stéphanie (2008) - Le matériel d'une boutique au sein de l'agglomération gallo-romaine du Vieil-Evreux (Eure). 2 vol. : 1-Mémoire, 166 p.; 2-Corpus. (dir. F. Dumasy et L. Guyard).

\section{Thèse de Doctorat}

Galinand, Cyrille (2008) - Usage social et symbolique du métal en France au Bronze ancien et moyen (dir. P. Brun).

\section{Université de Paris - EHESS}

\section{Thèse de Doctorat}

Langlois, Hugues (2008) - La basse vallée de Seine dans l'Antiquité, de Paris à l'estuaire. (dir. J. Andreau).

\section{Université de Paris 4}

Mémoire de Master 1

Bertaux, Valérie (2007) - Le matériel des sanctuaires des Aulerques Eburovices. (dir. M. Joly et L. Guyard).

\section{Université de Paris 6 - Pierre-et-Marie-Curie}

\section{Thèse de Doctorat}

Thiesson, Julien (2007) - Mesure et cartographie de la viscosité magnétique des sols. (91 p.) (dir. A. Tabbagh) [concerne Le Vieil-Évreux].

\section{Université du Maine (Le Mans)}

\section{Thèse de Doctorat}

Cormier, Sébastien 2008) - Les décors antiques de l'ouest de la Gaule. Synthèse sur l'architecture d'applique dans les territoires des Aulerques ( $f^{r}$ siècle-IIt siècle apr. J.-C.). 3 vol. : Texte (425+616 p.); Annexes et illustrations, (dir. R. Compatangelo-Soussignan et K. Gruel).

\section{UNIVERSITÉ DE ROUEN}

\section{Mémoires de Master 1}

Aubry, Émilie (2008) - Les possessions monastiques dans la forêt de Roumare durant la période ducale. (dir. A.-M. Flambard-Héricher et E. Lalou).

Gezolme, Guillaume (2007) - Mise en valeur du monument des eaux et de l'aqueduc gallo-romain du Vieil-Évreux (Eure). 66 p. (dir. G. Cachereul et L. Guyard).

LEDRET, Bénédicte (2008) - Bilan des connaissances sur le Néolithique final en Haute-Normandie. (dir. A.-M. Flambard-Héricher).

Roselier, Stéphane (2008) - L'empreinte des barbares dans le nord-ouest de la Gaule dans l'Antiquité tardive. (dir. S. Crogier-Pétrequin).

\section{Mémoire de Master 2}

Alizier, Juliane (2008) - La mise en valeur du grand sanctuaire de Gisacum (Vieil-Évreux - Eure). 160 p. (dir. L. Guyard). 